\title{
Mesure de matrices de transfert de systèmes acoustiques simples
}

\author{
V. DUBOS, J.-P. DALMONT et J. KERGOMARD \\ Laboratoire d'Acoustique, Université du Maine, BP. 535, 72017 Le Mans cedex, France
}

\begin{abstract}
The purpose of the present paper is to measure the transfer matrix of acoustical, reciprocal elements of wave guides by using two microphones and several terminal tubes, by mean of the least mean square method. We apply this method for the case of a lateral chimney. The results are satisfactory, but the accuracy is not large enough to determine the anti symmetrical field in the chimney.
\end{abstract}

\section{INTRODUCTION}

Les méthodes classiques de mesure de matrices de transfert impliquent quatre mesures de pressions, avec deux microphones de part et d'autre du système à étudier [1,2]. Un perfectionnement de ces méthodes consiste à utiliser deux charges terminales [3], ou deux sources symétriques $[4,5]$. Une autre amélioration consiste à employer un grand nombre de microphones ou un grand nombre de charges terminales. Citons également une méthode radicalement différente, pour mesurer l'effet de faibles discontinuités, basée sur une mesure fine d'un décalage de fréquence de résonance $[6,7]$.

Nous avons choisi d'utiliser une surdétermination du problème avec deux microphones et un grand nombre de charges terminales, et de traiter les données par une méthode de moindres carrés afin d'améliorer les résultats. Nous avons appliqué la mèthode de mesure à l'étude de l'effet d'une cheminée latérale symétrique, ouverte ou fermée, sur un guide d'ondes.

\section{PRESENTATION DE LA METHODE}

La matrice de transfert $(A, B, C, D)$ d'un quadripôle acoustique est définie par la relation suivante :

$$
\left(\begin{array}{l}
p \\
U
\end{array}\right)=\left(\begin{array}{ll}
A & C \\
B & D
\end{array}\right)\left(\begin{array}{l}
p_{1} \\
U_{1}
\end{array}\right)
$$

ou $\mathrm{p}$ et $\mathrm{p} /$ ont les pressions acoustiques, et $U$ et $U_{/}$les débits acoustiques aux extrémités du quadripôle considere.

Dans le cas d'un système réciproque, donc en particulier sans écoulement, la matrice de transfert peut ètre décrite par trois constantes complexes indépendantes, le quatrième coefficient étant dèterminé par la relation de réciprocité : $\mathrm{AD}-\mathrm{BC}=1$. Si, de plus, le système est symétrique, deux constantes seulement décrivent le système, car $A=D$. La matrice de transfert est alors déterminée par deux coefficients complexes, A et B par exemple. La mesure de ces coefficients peut être faite à l'aide de deux microphones placés de part et d'autre du guide étudié. Deux charges terminales connues ferment le système. L'emploi 
d'une surdétermination du problème avec un grand nombre de charges, et leur traitement par une méthode de moindres carrés permet d'améliorer les résultats.

\section{DISPOSITIF EXPERIMENTAL}

Un transducteur piézo-électrique $\mathrm{P}$ est alimenté par un générateur de fréquence pure (figure 1). Les signaux de pression acoustique, captés par deux microphones à électret $M$ et $M_{l}$, sont recueillis sur ordinateur par l'intermédiaire d'un voltmètre vectoriel. Le guide est désolidarisé de la source par l'insertion d'un morceau de chambre à air. On dispose de 6 tubes terminaux fermés (T5) de longueur et de diamètre connus.

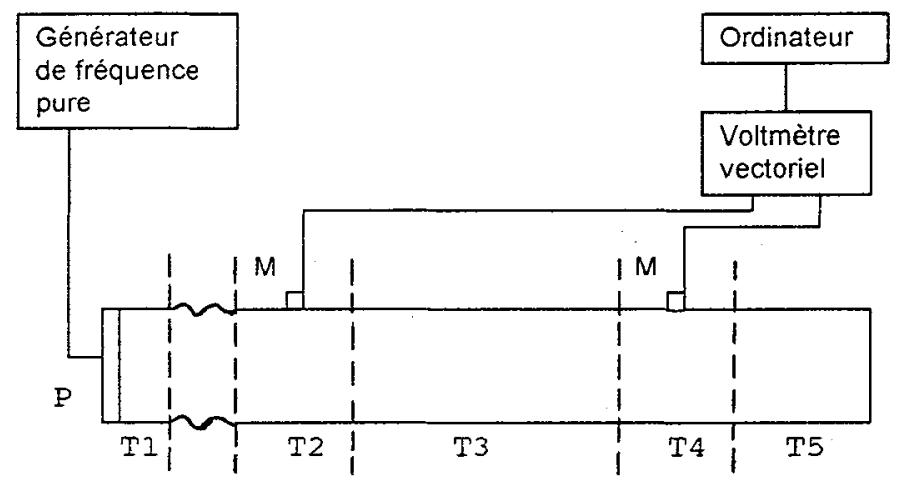

Figure 1 : dispositif expérimental pour la mesure de la matrice de transfert du guide $T 3$; les pointillés délimitemt les différents tubes: $M$ et $M l$ sont les deux microphones de mesure; $P$ est le transducteur piézo-électrique ; les tubes T2, T3 et T5 ont même section.

\section{UTILISATION DE LA METHODE DES MOINDRES CARRES}

Pour une charge $Z_{l, i}$, les deux équations reliant les pressions et débits acoustiques s'écrivent :

$$
\left\{\begin{array}{l}
\mathbf{p}_{i}=\mathbf{A} \cdot \mathbf{p}_{1, i}+\text { B. } U_{i, i} \\
U_{i}=C \cdot p_{t, i}+D \cdot U_{i, i}
\end{array}\right.
$$

où $\mathrm{p}_{\mathrm{i}}\left(\right.$ resp. $\mathrm{p}_{l, \mathrm{i}}$ ) et $\mathrm{U}_{\mathrm{i}}$ (resp. $\mathrm{U}_{l, i}$ ) représentent la pression et le débit acoustiques au niveau du premier microphone (resp. deuxième microphone). Le débit $U_{l, i}$ est relié à limpédance terminale par la relation :

$$
U_{1, i}=\frac{p_{1, i}}{Z_{1, i}}
$$

La determination de $\mathrm{A}$ et $\mathrm{B}$ est possible avec seulement deux charges terminales $\mathrm{i}$ et $\mathrm{j}$ à partir de la relation (1) et ne nécessite alors pas la connaissance de la source. Pour $n$ charges terminales, les coefficients A et B peuvent s'écrire, suivant un principe de moindres carrés [8]:

où

$$
A=\frac{\sum_{i<j} f_{i j}{ }^{*} \cdot g_{i j}}{\sum_{i<j}\left|f_{i j}\right|^{2}} \quad \text { et } \quad B=\frac{\sum_{i<j} f_{i j}{ }^{*} \cdot g^{\prime}{ }_{i j}}{\sum_{i<j}\left|f_{i j}\right|^{2}}
$$

ù :

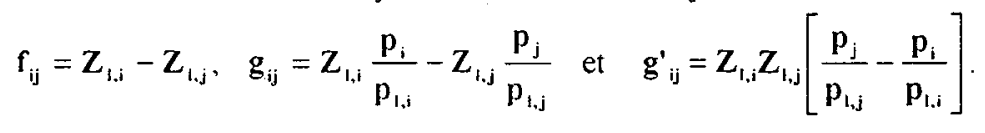


D'autres formes pour les termes $\mathrm{f}_{\mathrm{ij}}$, $\mathrm{g}_{\mathrm{ij}}$ et $\mathrm{g}_{\mathrm{ij}}^{\prime}$ ont été expérimentées, mais conduisent à des résultats sensiblement moins satisfaisants. Notons que la méthode des moindres carrés suppose des conditions de mesure identiques pour chaque tube terminal, ce qui est une condition difficile à réaliser expérimentalement, notamment en ce qui concerne la température.

\section{RESULTATS ET DISCUSSION}

\subsection{Matrice de transfert du tube étalon}

Seule la calibration des microphones relativement l'un à l'autre est nécessaire. Afin d'éviter leur démontage, nous préférons calibrer directement les microphones in situ. Le rapport de calibration est déterminé par le rapport des résultats expérimentaux et théoriques pour un tube étalon.

La figure 2 représente la partie réelle du premier coefficient de la matrice de transfert entre les deux microphones pour un tube étalon de longueur $0.517 \mathrm{~m}$ et de diamètre $20 \mathrm{~mm}$, déterminée à partir de deux et de six charges. L'erreur absolue sur le module de ce coefficient est en moyenne de 0.02 et au maximum de 0.1 .
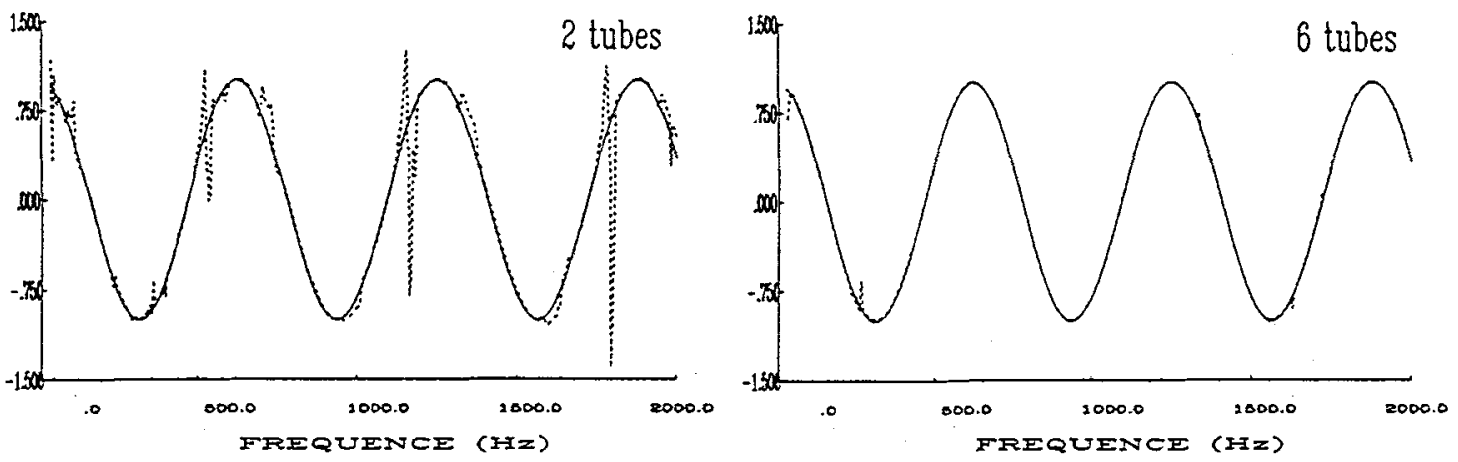

Figure 2 : partie réelle du prenier coefficient de la matrice de transfert, entre les deux microphones, d'un tube de longueur $0.517 \mathrm{~m}$ et de diamètre $20 \mathrm{~mm}$, déterminée à partir de deux tubes terminaux (2.a) et à partir de six tubes terminaux (2.b), en fonction de la fréquence ; théorie, ---- expérience.

La méthode des moindres carrés présente l'avantage de diminuer le bruit dû aux erreurs de mesure. Le résultat est nettement amélioré lorsque le nombre de tubes terminaux augmente. Ajoutons que la prise en compte d'un plus grand nombre de charges n'apporte pas d'amélioration notable.

\subsection{Matrice de transfert de cheminées latérales}

Une des motivations de ce travail est la mesure de matrices de transfert de guides surmontés de cheminées latérales ouvertes ou fermées. Le schéma électrique en $T$ équivalent aux basses fréquences à une cheminée latérale $[6,9]$ est constitué de deux inductances en série, représentant l'effet des modes antisymétriques dans la cheminée, et d'une branche en parallèle composée d'une impédance représentant l'effet du mode plan en série avec une inductance représentant l'effet des modes supérieurs.

Pour une cheminée latérale ouverte, aux basses fréquences, l'impédance en parallèle est une inductance dont la longueur équivalente est égale à la hauteur de la cheminée augmentée de la longueur équivalente à l'inductance représentant les modes supérieurs. La figure 3.a présente les résultats obtenus pour une cheminee de hauteur $7 \mathrm{~mm}$ et de diamètre $14 \mathrm{~mm}$. La méthode permet donc de déterminer la 
longueur équivalente d'une cheminée latérale. Par contre, l'effet des modes antisymétriques est trop faible pour être détecté (figure 3.b)
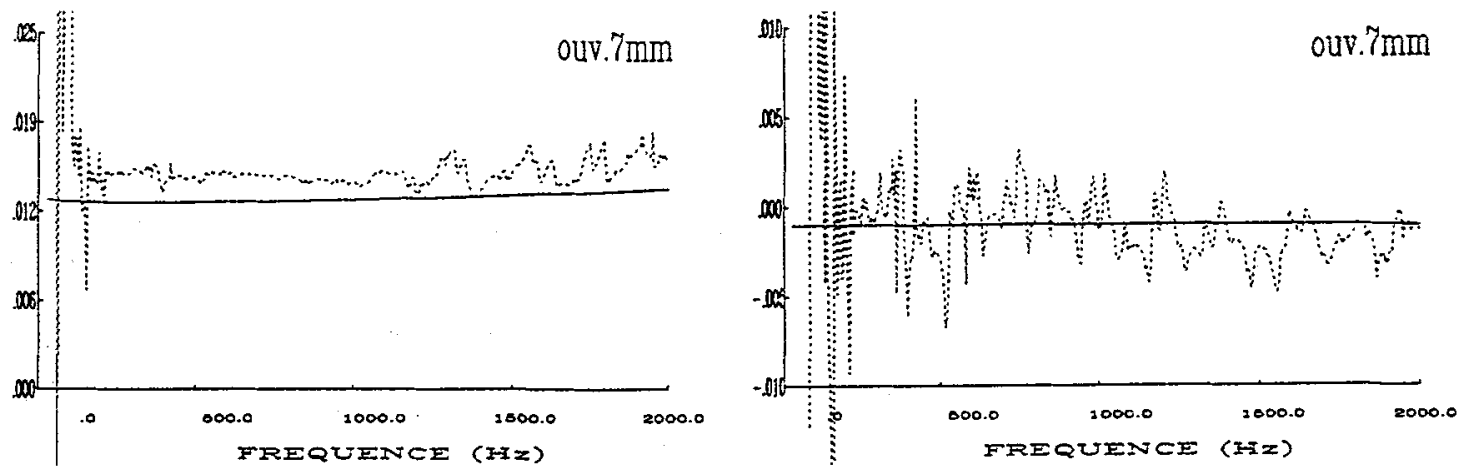

Figures 3 : longueurs équivalentes (en mètres) aux impédances en parallèle (3.a) et en série (3.b) du circuit électrique équivalemt d'une cheminée latérale ouverte de hauteur $7 \mathrm{~mm}$ et de diamètre $14 \mathrm{~mm}$, en fonction de la fréquence :

théorie, ----- expérience.

\section{CONCLUSION}

La méthode de mesure de matrices de transfert présentee démontre l'intérêt de la méthode des moindres carrés ; les résultats obtenus sont satisfaisants mais s'avèrent insuffisamment précis pour déterminer l'effet des modes antisymétriques dans une cheminée latérale. Une méthode basée sur des mesures de fréquences de résonance pourrait être, dans ce cas particulier, une alternative à cette méthode.

\section{REFERENCES}

[1] To C.W.S. and Doige G., J.Sound Vib. 62 (1979) 207-233.

[2] Lung T.Y. and Goige A.G., J.Acoust.Soc.Am. 73 (1983) 867-876.

[3] Nishimura M., Fukatsu S. and Akamatsu K., Proceedings of Imter-Noise 83 (1983) 395-398.

[4] Åbom M., Mechanical Systems and Signal Processing 5 (1991) 89-104.

[5] Abom M., Joumal of Sound and Vibration 155 (1992) 185-188.

[6] Keefe D.H., J.Acoust.Soc.Am. 72 (1982) 676-699.

[7]Dalmont J.P., Thèse de doctorat en Acoustique, 1988, Université du Maine, Le Mans, France.

[8]Desmons L. et Hardy J., to be published in J.Sound Vib.

[9] Kergomard J. et Khettabi A., J.Phys.IV France (1992) C1 85-88. 